\title{
A MATURIDADE DE UM CAMPO CIENTÍFICO: uma proposta metodológica a partir da Paleontologia brasileira
}

\author{
THE MATURITY OF A SCIENTIFIC FIELD: \\ a methodological proposition considering Brazilian Paleontology
}

\author{
Mell Longuinho André Siciliano \\ Jacqueline Leta $^{2}$
}

\begin{abstract}
RESUMO
0 desenvolvimento da ciência e suas dinâmicas de comunicação têm sido investigados através de técnicas oriundas da Bibliometria, Cientometria e Webmetria. 0 uso de tais abordagens permite uma diversidade de análises quantitativas da informação registrada a partir do fator de impacto, das citações, das autorias e coautorias, dentre outros aspectos. 0 objetivo do presente estudo é propor uma metodologia para estimar o grau de maturidade ou consolidação de um campo científico. A metodologia se baseia nas informações sobre autoria, mais especificamente o fluxo de autores ao longo do tempo. Para a aplicação desta metodologia, utilizou-se dados da Paleontologia brasileira, um campo de importância nacional que carece de estudos quantitativos que permitam entender seu atual grau de consolidação. A proposta tem quatro etapas principais, que inclui a definição da variável chave, as autorias, definição e coleta de dados da produção do campo, organização dos dados e análise das autorias. A partir da análises de autorias das publicações brasileiras em Paleontologia, indexadas na Web of Science, de 1991 a 2015, verificou-se que a quantidade de autores em dado espaço de tempo mostra expressivo e constante crescimento e que há um núcleo de autores que contribui de maneira contínua para o campo, com perspectiva de aumento, devido ao grande número de autores recém-chegados. A proposta mostrou-se não só viável para estimar a maturidade neste estudo de caso, mas também abre a possibilidade da realização de novos estudos, quer seja de acompanhamento dos avanços da maturidade deste campo no Brasil, bem como em outros campos do conhecimento.

Palavras-chave: Maturidade. Paleontologia. Bibliometria. Autoria. Ciência brasileira.
\end{abstract}

\section{ABSTRACT}

The development of science and its communication dynamics have been examined with the use of techniques from Bibliometrics, Scientometric and Webometrics. The use of such approaches allows a variety of quantitative analyzes of the information recorded, based on the impact factor, citations, authorship and co-authorship, among other aspects. The aim of the present study is to propose a methodology to estimate the degree of maturity or consolidation of a scientific field. The methodology is based on authorship information, more specifically the flow of authors over time. For the application of this methodology, data from Brazilian Paleontology was used, a field of national importance that lacks quantitative studies that allows scientists to understand its current degree of consolidation. The proposition has four main steps, which includes the definition of the key variable, namely authorship, definition and collection of data from the field production, data organization and analysis of the authorships. From the analysis of the authorships of Brazilian publications in Paleontology, indexed in Web Of Science, from 1991 to 2015, it was found that the number of authors in a given period of time shows expressive and constant growth and that there is a core of authors who contribute in a constant manner to the field, with a prospect of increase, due to the large number of newcomers. The proposition proved to be not only feasible to estimate the maturity in this case study, but also opens up the possibility of new applications, whether it is to monitor the advances in the maturity of this field in Brazil, as well as in other fields of knowledge.

Keywords: Maturity. Palaeontology. Bibliometrics. Authorship. Brazilian Science.

Artigo submetido em 24/04/2020 e aceito para publicação em 27/05/2020

1 Bibliotecária da Universidade Federal do Rio de Janeiro. Mestre em Ciência da Informação. Instituto Brasileiro de Informação em Ciência e Tecnologia, Brasil. Universidade Federal do Rio de Janeiro, Brasil. ORCID https://orcid.org/0000-0002-1096-4781. E-mail: mellsiciliano@gmail.com

2 Docente associada no Instituto de Bioquímica Médica. Universidade Federal do Rio de Janeiro, Brasil. ORCID https://orcid.org/00000002-3271-7749. E-mail: jleta@bioqmed.ufrj.br 


\section{INTRODUÇÃO}

Não é de hoje que pesquisadores se indagam sobre o processo de desenvolvimento da ciência e suas dinâmicas de comunicação. De fato, nos anos 1960, segundo Price (1963), já havia uma ampla discussão sobre o crescimento da ciência. Com o passar do tempo, as indagações se diversificaram, e pesquisadores passaram a estudá-la a partir de diferentes pontos de vista, seja da História da Ciência, da Ciência da Informação, da Sociologia, dentre tantas outras possibilidades. A verdade é que não há uma maneira única para analisar e compreender a ciência, seus atores, insumos ou produtos.

Na Ciência da Informaç̧ão, pesquisas voltadas para observação da comunidade científica e alguns processos intrínsecos à ciência, como o processo de comunicação cientifica, têm sido realizadas através de abordagens quantitativas, com técnicas da Bibliometria, Cientometria ou Webmetria. Tais técnicas permitem analisar quantitativamente a informação registrada e englobam estudos relativos a fator de impacto das publicações, análises de cocitação, estudos de autoria e coautoria, entre tantos outros.

Pesquisas com estas abordagens têm, como corpus de análise, majoritariamente documentos acadêmicos ou científicos (como artigos e livros) e também registros de patentes, ou seja, focam em documentos que retratam resultados tangíveis da pesquisa científica ou tecnológica. Tais estudos, com alguma frequência, se debruçam sobre campos específicos do conhecimento, como Neurociência (BRAUN; GLÄNZEL; SCHUBERT, 2001), Astrofísica (VELDEN; YAN; LAGOZE, 2017), Geologia (FIGUEIREDO, 1973) e Biologia (SUNDARARAJAN; PONNUDURAI, 2011), com fins de investigar questões específicas relacionadas ao desenvolvimento do referido campo, como a maturidade.

Investigações relacionadas à maturidade de um campo científico ocorrem pelo menos desde os anos 1980, e são, em sua maioria, operacionalizadas através de informações registradas nas publicações (KEATHLEY-HERRING et al., 2016). É importante, no entanto, destacar a falta de consenso sobre o conceito de maturidade. Isso se observa nas pesquisas, que utilizam diferentes definições para maturação, bem como diferentes abordagens para mensurar este conceito, conforme observado nos estudos de CHEON; GROVEN; SABHERWAL (1993), MALONI; CARTER; CARR (2009) e MENDES et al. (2017). Uma possível razão para a falta de consenso reside no fato de que os campos científicos não se consolidam de maneira previsível (KEATHLEY-HERRING et al., 2013). Na verdade, cada campo científico tem uma trajetória específica e um mesmo campo pode percorrer caminhos distintos de maturação em diferentes países e continentes.

Diante da diversidade de definições e indicadores para investigar a maturidade, o presente estudo selecionou alguns critérios apresentados no trabalho de Keathley-Herring et al. (2016) e, a partir 
destes, propõe uma metodologia a ser aplicada em estudos futuros que pretendam investigar o grau de maturidade ou a consolidação de um campo científico. Nesse trabalho, os autores realizaram um extensivo levantamento, mapeando nove dimensões e 24 critérios a partir dos quais é possível observar a maturidade de um determinado campo científico. A metodologia aqui apresentada foca na dimensão autoria que, para os autores, é a mais comumente aplicada em estudos com este foco; está intimamente associada ao fato de que se a publicação de documentos científicos é parte essencial da vida acadêmica, então seus autores, como parte da comunidade científica, são atores imprescindíveis, responsáveis não somente pela evolução intelectual de um campo científico, como também pela conquista de espaços de visibilidade e autonomia.

Para demonstrar a aplicabilidade desta metodologia, optou-se por mensurar a maturidade da Paleontologia brasileira, seja porque é um campo ainda pouco explorado do ponto de vista bibliométrico e cientométrico seja porque os estudos existentes não abordam a questão de maturidade ou consolidação do campo; como exemplo, há apenas o estudo de Miguel et al. (2013), intitulado Estudio bibliometrico de género en la paleontología de vertebrados. El caso de la revista argentina Ameghiniana (1957-2011). Já o foco na Paleontologia brasileira justifica-se por ser o Brasil um dos países que mais publicam em Paleontologia dentre os demais países da América Latina (SICILIANO, 2018), o que aponta para um papel protagonista na região.

Cabe ressaltar que a Paleontologia foi, durante muito tempo, entendida como uma subárea das Ciências Geológicas e, mais recentemente, também como uma subárea das Ciências Biológicas (HERBERT, 1985, KELLEY, 2013). Tal fato se reflete inclusive na formação profissional, considerando que em muitos países não existe um curso de graduação ou uma pós-graduação específica no campo. Em geral, a Paleontologia aparece como uma linha de pesquisa em programas de pós-graduação de outras áreas (ECHAORE-MCDAVID, 2008).

No Brasil, conforme tabela de classificação de áreas do conhecimento do Conselho Nacional de Desenvolvimento Científico e Tecnológico - CNPq e da Coordenação de Aperfeiçoamento de Pessoal de Nível Superior - Capes, ela aparece como Paleontologia Estratigráfica, uma subárea da Geociências, mas também como Paleobotânica e Paleozoologia, respectivamente subáreas da Botânica e da Zoologia (CONSELHO NACIONAL DE DESENVOLVIMENTO CIENTÍFICO E TECNOLÓGICO, 2020, COORDENAÇÃO DE APERFEIÇOAMENTO DE PESSOAL DE NÍVEL SUPERIOR, 2020).

Considerando este aspecto peculiar, é possível imaginar que a Paleontologia tenha sido abordada de maneira periférica e, de certa forma, invisibilizada em estudos bibliométricos que tratavam sobre Biologia ou Geologia, tal como no estudo realizado por Figueiredo (1973). 


\section{MATURIDADE DE UM CAMPO CIENTÍFICO}

A palavra maturidade, do latim maturitate, significa "Estado ou condição de maduro, de plenamente desenvolvido" (FERREIRA, 2010). Alguns estudos, como o de Rodrigues, Fonseca e Chaimovich (2000), utilizam, ao invés da palavra maturidade, a palavra consolidado para se referir ao desenvolvimento de um campo. Os termos, apesar de não serem exatamente sinônimos, estão relacionados, visto que a palavra consolidar, do latim consolidare, significa tornar sólido, seguro (FERREIRA, 2010).

Os estudos que visam medir a maturidade objetivam mensurar o nível de desenvolvimento e solidez de determinado campo científico, com especial interesse na criação, crescimento e disseminação do conhecimento (KEATHLEY-HERRING et al., 2016). Diversas são as abordagens de mensuração e também diversas são as concepções do que vem a ser um indicador de maturidade de um campo científico, podendo incluir índices que vão desde o crescimento da comunidade científica até o surgimento ou desaparecimento de métodos utilizados (CHEON; GROVEN; SABHERWAL, 1993; MALONI; CARTER; CARR, 2009; MENDES et al., 2017).

A falta de consenso sobre indicadores e abordagens dificulta a elaboração de um indicador universal para estimar ou medir a maturidade, aplicável a todos os campos científicos (CHEON; GROVEN; SABHERWAL, 1993, KEATHLEY-HERRING et al., 2016, MENDES et al., 2017). Mas um ponto consensual é que 0 amadurecimento de um campo científico é um fenômeno que necessariamente precisa de um espaço de tempo para ocorrer (CHEON; GROVEN; SABHERWAL, 1993). Por isso, estudos sobre maturidade, geralmente, debruçam-se sobre a literatura publicada em determinado campo científico, com enfoque nas mudanças ocorridas ao longo do tempo (KEATHLEY-HERRING et al., 2016).

Ao considerar as diversas pesquisas que abordam a maturidade de um campo científico, Keathley-Herring et al. (2016) levantaram que, de maneira geral, um campo científico é considerado maduro quando: a) é bem documentado e amplamente acessível, b) está estabelecido através de uma comunidade científica própria, c) é diferente de outros campos científicos, d) tem robustez de paradigmas, métodos e aplicações, e) causa impacto na comunidade científica (por exemplo, é citado por outros campos), e f) é colocado em prática (por exemplo, é aplicado na indústria).

Os autores ressaltam que alguns desses aspectos (como 0 uso fora do meio acadêmico) não são aplicáveis a todos os campos científicos e, por isso, os pesquisadores interessados em investigar a noção de maturidade devem escolher o(s) aspecto(s) mais apropriado(s) para a finalidade de suas pesquisas.

Keathley-Herring et al. (2016), em extensiva revisão da literatura, organizaram as abordagens já aplicadas em pesquisas sobre maturidade em nove dimensões de estudo, 24 critérios, e diversos 
subcritérios. Uma versão adaptada dos resultados apresentados pelos autores está no Quadro 1, onde é possível observar, as nove dimensões levantadas: Características de Autoria, Gênesis da Área, Características das Publicações, Características de Pesquisa, Características Teóricas, Características de Conteúdo, Impacto, Difusão e Infraestrutura. Cada uma destas dimensões possui pelo menos dois critérios associados, que descrevem um determinado aspecto da dimensão. Na dimensão Infraestrutura, por exemplo, os critérios associados são: Infraestrutura acadêmica e Infraestrutura de pesquisa. E cada critério, por sua vez, está associado a um ou mais subcritérios, que sinalizam a forma como o critério será operacionalizado. No critério Infraestrutura acadêmica, por exemplo, os subcritérios são Cursos Universitários e Programas de Pós-graduação.

Keathley-Herring et al. (2016) ainda ressaltam que enquanto alguns subcritérios são exclusivamente quantitativos ou qualitativos, em vários casos a operacionalização pode ser quantitativa e/ou qualitativa. Os subcritérios supracitados, por exemplo, podem ser analisados sob um prisma quantitativo (quantos são os Cursos Universitários e Programas de Pós-Graduação em determinado campo do conhecimento), como sob um prisma qualitativo (evolução da grade curricular destes cursos ao longo do tempo e possíveis influências de tais mudanças para a maturidade do campo em questão).

Quadro 1 - Dimensões e critérios de estudos de maturidade (continua)

\begin{tabular}{|c|c|c|}
\hline Dimensão & Critério & Subcritério \\
\hline \multirow{6}{*}{ Características de Autoria } & Quantidade de autores & $\begin{array}{c}\text { Autores existentes } \\
\text { Taxa de crescimento de autores }\end{array}$ \\
\hline & \multirow{3}{*}{ Diversidade de autores } & Disciplinas representadas \\
\hline & & Instituicóes representadas \\
\hline & & Paísẻs representados \\
\hline & \multirow{2}{*}{ Colaboração } & Colaboracões \\
\hline & & $\begin{array}{c}\text { Autorias múltiplas versus autorias únicas } \\
\text { Conexão entre autores }\end{array}$ \\
\hline \multirow{3}{*}{ Gênesis da Área } & \multirow[b]{2}{*}{ Primeiras publicações } & Tempo de vida da área \\
\hline & & $\begin{array}{l}\text { Consenso sobre primeiras publicacões } \\
\text { Caracteristicas das primeiras publicacóes }\end{array}$ \\
\hline & Teorias fundacionais & dentificacăo de teorias fundacionais \\
\hline \multirow{6}{*}{ Características das Publicações } & \multirow{2}{*}{$\begin{array}{l}\text { Quantidade de publicações } \\
\text { Publicacões como resultados }\end{array}$} & $\begin{array}{c}\text { romle de leonas iundacionals } \\
\text { Publicacóes }\end{array}$ \\
\hline & & Tendências de publicacões \\
\hline & Publicações como resultados & Resultados existentes \\
\hline & \multirow{3}{*}{ Referências } & $\begin{array}{l}\text { Quantidade de referências } \\
\text { Tempo de vida de referências }\end{array}$ \\
\hline & & Referências mais utilizadas \\
\hline & & Concentracão de referências \\
\hline \multirow{9}{*}{ Características da Pesquisa } & \multirow{3}{*}{ Métodos } & Métodos utilizados \\
\hline & & $\begin{array}{l}\text { Metodos mistos } \\
\text { Riaor na análise }\end{array}$ \\
\hline & & Abordagem \\
\hline & \multirow{3}{*}{ Rigor } & Claridade nos objetivos de pesquisa \\
\hline & & Confiabilidáde e validáde \\
\hline & & $\begin{array}{c}\text { Rigor estatístico } \\
\text { Exaustividade }\end{array}$ \\
\hline & \multirow{3}{*}{ Variáveis } & Conexão entre estudos \\
\hline & & Tipos de variáveis representadas \\
\hline & & Tipo de finalidade (teórica ou aplicada) \\
\hline
\end{tabular}

Fonte: Adaptado de KEATHLEY-HERRING et al., 2016 
Quadro 1 - Dimensões e critérios de estudos de maturidade (continua)

\begin{tabular}{|c|c|c|}
\hline Dimensão & Critério & Subcritério \\
\hline \multirow{4}{*}{ Características Teóricas } & \multirow[b]{2}{*}{$\begin{array}{l}\text { Desenvolvimento de novas } \\
\text { teorias }\end{array}$} & Desenvolvimento de modelos \\
\hline & & $\begin{array}{c}\text { Publicações voltadas para a construção de } \\
\text { teorias }\end{array}$ \\
\hline & \multirow{2}{*}{ Uso de teorias existentes } & Fontes de teorias \\
\hline & & Aplicação de teorias \\
\hline \multirow{11}{*}{ Características de Conteúdo } & \multirow{4}{*}{ Temas } & Temas abordados \\
\hline & & Conexão entre temas \\
\hline & & Estabilidade de temas \\
\hline & & Consistência em citações sobre um tema \\
\hline & \multirow{3}{*}{ Escopo } & Objeto de estudo \\
\hline & & Indicação de estudos futuros \\
\hline & & Implicações práticas \\
\hline & \multirow{4}{*}{ Tópicos } & Desenvolvimento de subcampos \\
\hline & & Consistência terminológica \\
\hline & & Palavras-chave utilizadas \\
\hline & & Conexão entre palavras-chave \\
\hline \multirow{7}{*}{ Impacto } & \multirow{2}{*}{ Impacto de autores } & Ranking institucional \\
\hline & & Produtividade \\
\hline & \multirow{5}{*}{ Impacto de publicações } & Ranking de publicações \\
\hline & & Concentração de publicações \\
\hline & & $\begin{array}{c}\text { Existências de publicações fundamentais (que } \\
\text { geraram várias outras pesquisas) }\end{array}$ \\
\hline & & Citações \\
\hline & & Cocitações \\
\hline \multirow{10}{*}{ Difusão } & \multirow{3}{*}{ Aplicabilidade na indústria } & $\begin{array}{l}\text { Posições formais em instituições não } \\
\text { acadêmicas }\end{array}$ \\
\hline & & Resultados práticos (guias, manuais, etc.) \\
\hline & & Serviços de consultoria \\
\hline & \multirow{4}{*}{ Comunidades de prática } & Eventos \\
\hline & & Sociedade profissionais \\
\hline & & Comunidades online \\
\hline & & Fóruns de discussão \\
\hline & \multirow{3}{*}{ Desenvolvimento de tecnologias } & Produtos comerciais \\
\hline & & Patentes \\
\hline & & Aplicação prática de teorias \\
\hline
\end{tabular}

Fonte: Adaptado de KEATHLEY-HERRING et al., 2016

Quadro 1 - Dimensões e critérios de estudos de maturidade

\begin{tabular}{|c|c|c|}
\hline Dimensão & Critério & Subcritério \\
\hline \multirow{4}{*}{ Infraestrutura } & \multirow{2}{*}{ Infraestrutura acadêmica } & Cursos universitários \\
\cline { 3 - 3 } & & Programas de pós-graduação \\
\cline { 2 - 3 } & \multirow{3}{*}{ Infraestrutura de pesquisa } & Organismos de certificação \\
\cline { 3 - 3 } & & Programas de financiamento \\
\cline { 3 - 3 } & & Centros de pesquisa \\
\hline
\end{tabular}

Fonte: Adaptado de KEATHLEY-HERRING et al., 2016 
Dentre as nove dimensões mapeadas, a dimensão Característica de Autoria é a mais utilizada em estudos sobre maturidade (KEATHLEY-HERRING et al., 2013). A seguir uma breve descrição de alguns dos estudos que se basearam nesta dimensão.

Rodrigues, Fonseca e Chaimovich (2000) abordaram a maturidade sob o ponto de vista do crescimento de autores e da permanência ou não de autores, dentre os anos 1981-1995, nos seguintes campos científicos, no Brasil: câncer, doenças cardiovasculares e malária. Os resultados indicaram maturidade do campo de pesquisa doenças cardiovasculares, enquanto os campos de pesquisa câncer e malária estariam ainda em fase crescimento, mas com avançado estágio de maturação.

MALONI; CARTER; CARR (2009), através de características de autoria, testaram o nível de maturação do campo Logística, cobrindo um período de 16 anos de pesquisa. Os autores compararam os resultados com os de campos correlatos, como Contabilidade, Finanças e Marketing, chegando à conclusão de que, comparativamente, o campo Logística está ainda em fase de maturação, apesar de apresentar constante crescimento ao longo dos anos estudados.

Koseoglu (2016) focou nas colaborações entre autores no campo Planejamento Estratégico, tendo como corpus de análise artigos publicados no periódico Strategic Management Journal, dentre os anos 1980 a 2014. Os resultados indicaram baixo nível de maturação do campo estudado.

Por fim, Mendes et al. (2017) estudaram o campo de pesquisa Desenvolvimento de Serviços e aplicaram diversas dimensões em seu estudo, comparando-as entre si. No que se refere à autoria, os autores focaram em redes de coautoria, com destaque para a centralidade de grau e conexão entre autores. Os resultados do estudo indicaram que o campo de pesquisa está em fase de maturação, com diferentes níveis de maturidade em cada uma das dimensões estudadas. Por este motivo, os autores afirmaram que a conclusão de que um campo está totalmente maduro pode ser uma afirmativa enganosa.

0 extenso estudo de Keathley-Herring et al. (2013) assim como a literatura ainda escassa acerca dos indicadores de maturidade de um campo científico sugerem uma lacuna teórico metodológica sobre como tratar esta temática. A dimensão autoria, devido à disponibilidade e ao acesso relativamente fácil a tais informações, é uma forte candidata a integrar propostas metodológicas sobre a maturidade dos mais diversos campos do conhecimento. É esta a dimensão escolhida para o presente estudo. A proposta metodológica e etapas estão detalhadas a seguir. 


\section{A PROPOSTA METODOLÓGICA PARA MENSURAR A MATURIDADE DE UM CAMPO}

A proposta apresentada parte de uma etapa inicial de definição da variável chave, as autorias, e depois segue pela definição e coleta de dados da produção de um campo científico, organização dos dados e análise das autorias.

\subsection{Definindo as autorias}

A partir das categorias levantadas por Keathley-Herring et al. (2016) e dos critérios utilizados por Rodrigues, Fonseca e Chaimovich (2000) e Mendes et al. (2017), o presente estudo propõe a mensuração da maturidade da Paleontologia através das características de autoria, quais sejam: (a) crescimento no número de autores, que pode ser aferido através da contagem simples, e (b) a existência de autores chave, ou seja, permanentes, o que pode ser aferida através do Esquema de Fluxo de Autoria, de Price e Gürsey (1975).

Price e Gürsey (1975) observaram que, em determinado intervalo de tempo, um grande número de autores em um campo são transitórios, ou seja, nunca apareceram antes e nunca aparecerão outra vez; em contrapartida, uma parte menor dos autores pode ser considerada como estável. Os pesquisadores estabeleceram, então, quatro categorias de autores: (a) Newcomers (recém-chegados ${ }^{3}$ ), (b) Continuants (permanentes), (c) Transients (transientes) e (d) Terminators (terminais). Tais categorias foram estabelecidas utilizando um ano específico como referência.

Na Figura 1, é possível ver o esquema do fluxo de autoria de Price e Gürsey (1975), onde estão indicadas algumas situações: (i) Autores Nunca antes que continuam Para o próximo ano e Para anos posteriores são os Newcomers (recém-chegados ${ }^{4}$ ), (ii) Autores Do último ano e De anos anteriores que continuam Para o próximo ano e Para anos posteriores são os Continuants (permanentes), (iii) Autores Nunca antes e Nunca mais são os Transients (transientes) e (iv) Autores Do último ano e De anos anteriores que seguem para Nunca mais são os Terminators (terminais). Resumidamente, os autores permanentes seriam aqueles que aparecem em todos os anos, de maneira ininterrupta.

3 Tradução nossa.

4 Ser um autor recém-chegado não significa ser um pesquisador recém-formado, significa tão somente que aparece pela primeira vez como autor no grupo de publicações estudado. 
Figura 1 - Esquema do fluxo de autoria

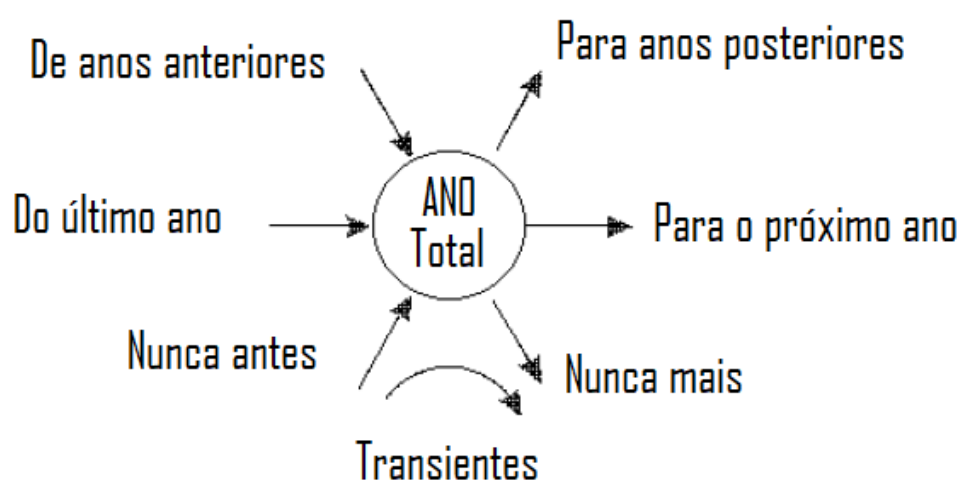

Fonte: Adaptado de Price e Gürsey, 1975.

\subsection{Coletando os dados de autoria}

A Web of Science/Clarivate Analytics - WoS ${ }^{5}$ foi a base de dados escolhida para a coleta dos dados da produção cientifica da Paleontologia brasileira, em especial das autorias. Importante destacar a WoS é uma base de dados referencial interdisciplinar, que indexa 33 mil periódicos, de diversas áreas do conhecimento, abrangendo 256 disciplinas, e com cobertura temporal que, de maneira geral, vai desde 1900 até a atualidade (CLARIVATE ANALYTICS, 2017). Segundo Costas (2017), a WoS é a base de dados mais utilizada em estudos cientométricos. 0 principal atrativo é a grande diversidade de metadados indexados, que incluem, além das informações bibliográficas básicas (título, ano de publicação, nome do periódico, volume, etc), informações como nome dos autores, filiação, citações e, mais recentemente, dados sobre financiamento e agradecimentos (COSTAS, 2017). A base também permite que os dados das publicações sejam baixados facilmente, em sua completude.

Uma vez definida a fonte de dados, a coleta foi realizada em 04 de maio de 2017, na Coleção Principal da WoS. Os dados foram obtidos através da seguinte estratégia de busca: na modalidade Busca Avançada, no campo Categoria (código WC), usou-se o termo Paleontology, com a finalidade de recuperar todas as publicações que estivessem enquadradas nessa categoria. A seguir, aplicou-se um filtro no campo Tempo Estipulado, onde foi inserido o intervalo de 1991 até 2015.

Cabe ressaltar que a WoS é a única, dentre as principais bases de dados referenciais disponíveis, que contém a categoria Paleontology como opção de pesquisa nas categorias de assunto. Sua existência

5 Disponível através do Portal Capes. 
permite a realização de uma pesquisa temática, cujo resultado apresenta somente as publicações classificadas nesta categoria ${ }^{6}$.

Após o resultado, aplicou-se um segundo filtro, no campo Países/Territórios, que refina a pesquisa considerando a filiação dos autores das publicações, para restringir apenas aos documentos publicados por autores filiados a instituições brasileiras.

0 Quadro 2 apresenta a estratégia de busca final para o levantamento dos dados, com os filtros inclusos.

Quadro 2 - Estratégia de busca aplicada na WoS para a obtenção dos dados de pesquisa.

\section{Categoria de assunto (WC): PALEONTOLOGY}

País/Região: (BRAZIL)

Tempo estipulado: de 1991 a 2015

Fonte: Elaboração dos autores.

\subsection{Organizando e categorizando as autorias}

Para esta etapa, o primeiro passo foi a normalização manual de nomes de autores e instituições. As grandes bases de dados da produção cientifica mundial, como a WoS, apresentam uma boa qualidade de estruturação de metadados se comparadas as demais; mas os dados de autoria ainda requerem uma padronização extensiva (SMALHEISER; TORVIK, 2009, COSTA, 2017), devido ao grande número de entradas diferentes atribuídas a uma mesma instituição ou um mesmo autor.

Segundo Smalheiser e Torvik (2009), no que tange às autorias, os casos mais comuns são: erros de digitação (exemplo deste estudo, MIGOTT, R em vez de MIGOTTO, R), variantes do nome (exemplo deste estudo, KELLNER, AW e KELLNER, A), mudanças do nome adotado pelo autor ao longo

6 A base de dados Scopus, principal concorrente da WoS, apesar de também classificar seus periódicos em categorias, permite apenas que sejam feitas pesquisas por grandes categorias, no caso Earth and Planetary Sciences. Nesta base a categoria Paleontology é uma subcategoria de Earth and Planetary Sciences. 
do tempo (exemplo deste estudo, SILVA, VG nos primeiros períodos e GALLO, V nos últimos períodos), e homônimos (exemplo deste estudo, CARVALHO, M se referindo a Carvalho, Marcelo e também a Carvalho, Maria). 0 processo é trabalhoso pois muitos casos envolvem a consulta às publicações, principalmente com os homônimos.

Assim, para esta etapa de normalização dos dados de autoria, foi gerado um tesauro no programa Microsoft Excel, contendo 200 entradas ambíguas de autor, ou seja, duas ou mais entradas que se referiam ao mesmo autor.

Concluída esta etapa, procedeu-se a identificação dos autores chave, isto é, aqueles que publicam de maneira contínua no campo. Considerando que a presente análise cobre 25 anos de pesquisa em Paleontologia, estes foram divididos em cinco quinquênios (1991-1995, 1996-2000, 2001-2005, 20062010 e 2011-2015) e o período central (2001-2005) foi usado como o período base para a identificação dos autores permanentes.

Esta etapa também foi executada no programa Microsoft Excel, através do uso dos comandos de filtro. Assim foi possível classificar os 169 autores do período base nas quatro categorias préestabelecidas (recém chegados, permanentes, transientes e terminais).

\subsection{Medindo a maturidade a partir das autorias}

Considerando a estratégia apresentada no Quadro 2, foram recuperadas, no total, 994 publicações com ao menos um autor filiado ao Brasil. Entende-se, portanto, que no presente trabalho, produção científica em Paleontologia refere-se somente àquelas publicações cujas fontes estão indexadas na categoria Paleontology da WoS; em outras palavras, o corpus de análise inclui somente publicações de periódicos especializados em Paleontologia. Importante ressaltar que as análises não fazem distinção entre 0 tipo de publicação, ou seja, levam em conta artigos, resumos de congresso, revisões, entre outros.

Em relação ao crescimento no número de autores, a Tabela 1 aponta que houve não só crescimento no número de autores, como também crescimento na produção nacional em Paleontologia. Em números absolutos, no primeiro período havia 38 autores e no último 647, ou seja, um crescimento de $1.602,6 \%$. Já quanto aos artigos, no primeiro período havia 25 , e último 465 , um crescimento de $1.760 \%{ }^{7}$.

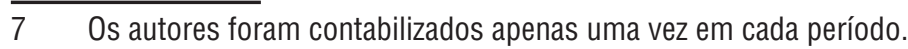


Tabela 1 - Crescimento da produção brasileira e de autores em Paleontologia, na Web of Science, entre 1991-2015.

\begin{tabular}{|l|c|c|}
\hline Período & N. Artigos & N. Autores \\
\hline $1991-1995$ & 25 & 38 \\
\hline $1996-2000$ & 72 & 84 \\
\hline $2001-2005$ & 145 & 169 \\
\hline $2006-2010$ & 287 & 333 \\
\hline $2011-2015$ & 465 & 647 \\
\hline Total & 994 & 1.271 \\
\hline
\end{tabular}

Fonte: Dados de pesquisa.

Já em relação a existência de autores chave, isto é, aqueles que publicam de maneira contínua no campo, foi aplicado o já mencionado esquema do fluxo de autoria de Price e Gürsey (1975). Como a presente análise cobre 25 anos de pesquisa em Paleontologia, divididos em cinco períodos, o período base para a identificação dos autores permanentes é o período central, isto é, 2001-2005. Neste período, há um total de 169 autores, que se distribuem em quatro tipos de autores, conforme Tabela 2.

Tabela 2 - Tipos de autores filiados ao Brasil em publicações em Paleontologia, na Web of Science, entre 1991-2015, segundo o esquema do fluxo de autoria de Price e Gürsey (1975).

\begin{tabular}{|l|c|c|}
\hline Tipo de autor & Total de autores & $\%$ \\
\hline Transientes & 63 & 37,3 \\
\hline Recém-chegados & 60 & 35,5 \\
\hline Permanentes & 37 & 21,9 \\
\hline Terminais & 9 & 5,3 \\
\hline Total de autores no período & 169 & 100 \\
\hline
\end{tabular}

Fonte: Dados de pesquisa, utilizando como período base 2001-2005.

A quantidade de autores transientes representa a maior parte dos autores (37,3\%), seguindo o padrão encontrado em estudos anteriores, como os de Price e Gürsey (1963) e Braun, Glänzel e Schubert (2001). Os autores recém-chegados representam o segundo maior contingente de autores (35,5\%), o que pode ser interpretado como um sinal positivo; visto que tais autores continuam a aparecer em períodos posteriores, há a possibilidade de, no futuro, fazerem parte do grupo de autores considerados permanentes. 0 grupo de recém-chegados, portanto, tem um potencial de se inserir definitivamente no campo e, assim, desenvolver pesquisas de maneira constante. Apesar do grande 
número de autores entrando no sistema, poucos são aqueles que saíram do sistema, ou seja, terminais. Este grupo de autores representa somente $5,3 \%$ do total.

Quanto aos autores permanentes, ou seja, aqueles que aparecem no período base, nos períodos anteriores e nos períodos subsequentes, mesmo que haja algum intervalo entre os períodos, eles representam 21,9\% do total, o que indica que a Paleontologia brasileira possui um número expressivo de pesquisadores que se dedicam de maneira contínua na área, ainda que não seja de maneira ininterrupta. É possível, ainda, separar os autores permanentes em dois grupos, de acordo com a regularidade de publicação, sem interrupção ou com interrupção na produção, conforme Tabela 3.

Tabela 3 - Autores permanentes filiados ao Brasil em Paleontologia, na Web of Science, entre 19912015, divididos entre atividade sem interrupção e atividade com interrupção, de acordo com o esquema do fluxo de autor de Price e Gürsey (1975).

\begin{tabular}{|l|c|c|}
\hline Tipo de autor & Total de autores & $\%$ \\
\hline Permanentes - Atividade sem interrupção & 7 & 18,9 \\
\hline Permanentes - Atividade com interrupção & 30 & 81,1 \\
\hline Total de autores permanentes no período & 37 & 100 \\
\hline
\end{tabular}

Fonte: Dados de pesquisa, utilizando como período base 2001-2005.

Do total de autores permanentes, apenas sete $(18,9 \%)$ têm atividade sem interrupção. A maioria $(81,1 \%)$ publica com interrupções, ou seja, não publicam em todos os períodos. Como ressaltado por Gordon (2007), a existência autores que publicam de maneira constante demonstra o comprometimento desses pesquisadores com o campo; mesmo que essa atividade seja com interrupções, que podem ocorrer, vale lembrar, por inúmeros fatores. No presente estudo um desses fatores poderia ser, por exemplo, ter publicado em determinado período em periódico não indexado pela WoS.

Sobre este grupo de autores, Braun, Glänzel e Schubert (2001) apontaram que estes são, frequentemente, responsáveis por fazer a ponte com as outras categorias de autoria, ou seja, os autores recém-chegados, transientes e terminais aparecem, majoritariamente, em coautoria com os autores permanentes de um campo. Uma das razões para isso é que tais autores, por terem uma posição estável no campo em que atuam, detêm, de maneira geral, mais conhecimento e recursos que os demais autores (WAGNER; LEYDESDORFF, 2005).

Price e Gürsey (1975) ressaltam ainda a existência de autores que não aparecem no período base, mas que apareceram nos períodos anteriores e também nos subsequentes. Em tal situação, os 
autores fariam parte do grupo de autores permanentes com atividade irregular, que saltaram justamente o período base. No presente estudo existem 15 autores enquadrados neste caso.

\section{CONSIDERAÇÕES FINAIS}

Investigar um campo científico, qualquer que seja, não é uma tarefa fácil. São muitas as abordagens disponíveis e a adequação de cada uma depende do campo científico estudado. Porém, no que tange à mensuração da maturidade de um campo científico, a proposta baseada nas autorias apresenta vantagens, visto que (1) as análises de autoria já foram apontadas como a abordagem mais comum em estudos de maturidade (KEATHLEY-HERRING et al., 2013), (2) os autores são peças-chave no crescimento de qualquer campo científico, o que potencializa a aplicação da metodologia em outros campos e (3) o acesso relativamente fácil a estas informações.

A aplicação da proposta metodológica a partir de um caso, a produção brasileira em Paleontologia, sugere que a Paleontologia brasileira atende de maneira integral ou parcial aos indicadores de maturidade do ponto de vista das características de autoria. Esta constatação tem como base (a) a quantidade de autores em dado espaço de tempo, o primeiro subcritério do estudo de KEATHLEY-HERRING et al. (2013), que mostra expressivo e constante crescimento no número de autores (Tabela 1) e (b) a ocorrência de 37 autores permanentes, segundo análise baseada no Esquema de Fluxo de Autoria de Price e Gürsey (1975), ou seja, um núcleo de autores que contribui de maneira contínua para o campo (Tabelas 2 e 3). Importante destacar ainda a perspectiva de aumento desde núcleo, devido ao grande número de autores recém-chegados, que representam $35,5 \%$ do total.

Considerando o exposto, pode-se inferir que, se a Paleontologia brasileira ainda não está em um estágio pleno de maturação, os resultados indicam que ela já possui um grau avançado de maturidade. A existência de um núcleo dedicado, por um lado, e o crescimento no número de autores, por outro, são sinais de que - do ponto de vista do capital intelectual - o campo tem potencial para expandir e para se consolidar como um dos principais expoentes da Paleontologia Latino-Americana.

A proposta apresentada aqui mostrou ser capaz e viável de caracterizar e avaliar a maturidade de um campo científico, em um dado momento da história e em uma dada localidade geográfica. A proposta, portanto, abre não apenas a possibilidade da realização de estudos futuros para acompanhar os avanços da maturidade deste campo no Brasil, como também traz uma sistematização que pode ser apropriada e utilizada em outros campos do conhecimento. 


\section{REFERÊNCIAS}

BRAUN, T.; GLÄNZEL, W.; SCHUBERT, A. Publication and cooperation patterns of the authors of neuroscience journals. Scientometrics, [Dordrecht], v. 50, n. 3, p.499-510, 2001.

CHEON, M. J.; GROVEN, V.; SABHERWAL, R. The evolution of empirical research in IS: a study in IS maturity. Information \& Management, [s.I.], v. 24, n. 3, p.107-119, mar. 1993.

CLARIVATE ANALYTICS. Web of Science: trust the difference. 2017. Disponivel em: <https://clarivate. com/products/web-of-science/\#regional>. Acesso em: 11 jul. 2017.

CONSELHO NACIONAL DE DESENVOLVIMENTO CIENTÍFICO E TECNOLÓGICO. Tabela de

Áreas do Conhecimento. Disponível em: http://www.cnpq.br/documents/10157/186158/

TabeladeAreasdoConhecimento.pdf. Acesso em: 06 maio 2020.

COORDENAÇÃO DE APERFEIÇOAMENTO DE PESSOAL DE NÍVEL SUPERIOR. Tabela de Áreas do Conhecimento. Disponível em: http://www.capes.gov.br/images/stories/download/avaliacao/ TabelaAreasConhecimento_042009.pdf. Acesso em: 06 maio 2020.

COSTAS, R.. Discussões gerais sobre as características mais relevantes de infraestruturas de pesquisa para a cientometria. Bibliometria e Cientometria no Brasil: infraestrutura para avaliação da pesquisa científica na Era do Big Data, p. 19-42, 2017.

ECHAORE-MCDAVID, S.. Paleontologist. In: ECHAORE-MCDAVID, S.. Career Opportunities in Science. 2. ed. New York: Ferguson, 2008. p. 87-89.

FERREIRA, A. B. H.. Mini Aurélio: o dicionário da língua portuguesa. Curitiba: Positivo, 2010.

FIGUEIREDO, L. M. . Distribuição da Literatura Geológica Brasileira: Estudo Bibliométrico. Ciência da Informação, Rio de Janeiro, v. 2, n. 1, p.27-40, 1973.

GORDON, A.. Transient and continuant authors in a research field: The case of terrorism.

Scientometrics, [Dordrecht], v. 72, n. 2, p.213-224, 17 jun. 2007.

HERBERT, S.. Darwin the young geologist. In: KOHN, David (Ed.). The Darwinian heritage. New Jersey: Princeton University Press, 1985. Cap. 16, p. 504.

KEATHLEY-HERRING, H. et al. Proposed Maturity Assessment Framework for a Research Field. In: INDUSTRIAL AND SYSTEMS ENGINEERING RESEARCH CONFERENCE, 2013, San Juan.

Proceedings... . San Juan, 2013.

KEATHLEY-HERRING, H. et al. Assessing the maturity of a research area: bibliometric review and proposed framework. Scientometrics, [Dordrecht], v. 109, n. 2, p.927-951, 2016.

KELLEY, P. H. et al. From paleontology to paleobiology: a half-century of progress in understanding life history. In: BICKFORD, Marion E. The Web of Geological Sciences: advances, Impacts, and Interactions, Colorado: Geological Society of America, 2013. p.191-232. 
KOSEOGLU, M. A.. Growth and structure of authorship and co-authorship network in the strategic management realm: Evidence from the Strategic Management Journal. Business Research Quarterly, [s.l.], v. 19, n. 3, p.153-170, jul. 2016.

MALONI, M. J.; CARTER, C. R.; CARR, A. S.. Assessing logistics maturation through author concentration. International Journal of Physical Distribution \& Logistics Management, [s.I.], v. 39, n. 3, p.250-268, 17 abr. 2009.

MENDES, G.H.S. et al. Uncovering the structures and maturity of the new service development research field through a bibliometric study (1984-2014). Journal of Service Management, [s.I.], v. 28, n. 1, p.182-223, 20 mar. 2017.

MIGUEL, S. et al. Estudio bibliométrico de género en la paleontología de vertebrados. El caso de la revista argentina Ameghiniana (1957-2011). Investigación Bibliotecológica, Cidade do México, v. 29, n. 61, p.133-155, set./dez. 2013.

PRICE, D. J. S.. Little Science, Big Science. Nova York: Columbia University Press, 1963.

PRICE, D. J. S.; GÜRSEY, S. Studies in scientometrics i: transience and continuance in scientific authorship. Ciência da Informação, v. 4, n. 1, p. 27-40, 1975.

RODRIGUES, P.S; FONSECA, L.; CHAIMOVICH, H. Mapping cancer, cardiovascular and malaria research in Brazil. Brazilian Journal of Medical and Biological Research, [s.I.], v. 33, n. 8, p.853867, ago. 2000.

SICILIANO, M. L. A. Paleontologia brasileira: uma análise sob o ponto de vista da maturidade. 2018. 107 f. Dissertação (Mestrado) - Curso de Ciência da Informação, Universidade Federal do Rio de Janeiro; Instituto Brasileiro de Informação em Ciência e Tecnologia, Rio de Janeiro, 2018.

SMALHEISER, N. R.; TORVIK, V. I. Author name disambiguation. Annual Review of Information Science And Technology, [s.I.], v. 43, n. 1, p.1-43, 2009.

SUNDARARAJAN, D.; PONNUDURAI, R. Bibliometric study of marine biology publication output at international level. International Journal of Recent Scientific Research, [s. I.], v. 2, n. 11, p.276-278, nov. 2011.

VELDEN, T.; YAN, S.; LAGOZE, C. Mapping the cognitive structure of astrophysics by infomap clustering of the citation network and topic affinity analysis. Scientometrics, [Dordrecht], v. 111, n. 2, p.1033-1051, 27 fev. 2017.

WAGNER, C. S.; LEYDESDORFF, L. Network structure, self-organization, and the growth of international collaboration in science. Research Policy, [s.l.], v. 34, n. 10, p.1608-1618, dez. 2005. 